Journal of Mathematics and Statistics 8 (1): 42-48, 2012

ISSN 1549-3644

(C) 2012 Science Publications

\title{
A Comparison of the Bayesian and Other Methods for Estimation of Reliability Function for Burr-XII Distribution
}

\author{
Safaa Ali Nasir and Nashaat Jaisam Al-Anber \\ Department of Information Technology, \\ Technical College of Management-Baghdad, \\ Foundation of Technical Education, Iraq
}

\begin{abstract}
Problem statement: The Burr-XII distribution has been widely used especially in the modeling of life time event data. It provides a statistical model which has a wide variety of application in many areas and the main advantage is its ability in the context of life time event among other distributions. The conventional maximum likelihood method is the usual way to estimate the parameters of a distribution. Bayesian approach has received much attention in contention with other estimation methods. In this study we explore and compare the performance of the maximum likelihood and median estimates with the Bayesian estimate of Reliability function for the two parameter BurrXII distribution. Approach: The maximum likelihood estimation, median estimation, Bayesian using Jeffrey prior, modified Jeffery prior and extension of Jeffery prior information for estimation the Reliability function of Burr-XII distribution of life time are presented. We explore the performance of these estimators numerically under varying conditions. Through the simulation study a comparison are made on the performance of these estimators with respect to the Integrated Mean Square Error (IMSE) and Integrated Mean Absolute Percentage Error (IMAPE). Results: For all the varying sample size, several values of the shifting parameter for the two parameter Burr-XII distribution and for the values for the extension of Jeffery prior, the estimator of Bayesian methods result in smaller IMSE compared to the classical methods in majority of the cases especially in small size of shape parameter. Also in all cases for both methods the IMSE and IMAPE decreases as sample size increases. Conclusion: Based on the results of this simulation study the Bayesian approach used in the estimating of reliability function for the two parameter Burr-XII distribution is found to be superior compared to the conventional methods with respect to IMSE values.
\end{abstract}

Key words: Extension of Jeffery prior information, Burr-XII distribution, Bayes method

\section{INTRODUCTION}

Reliability theory is mainly concerned with the determination of the probability that a system, consisting possibly of several components, will operate adequately for a given period of time in its intended application. The Reliability function $\mathrm{R}(\mathrm{t})$ is defined as the probability that system will operate at time period $\mathrm{t}$. The two parameter Burr-XII distribution was first introduced in the literature by Burr (1942) and has gained special attention in the last two decades due to the importance of using it in practical situations especially in reliability studies and failure time modeling since the Burr-XII distribution has a

Non-monotone hazard function, which can accommodate many shapes of hazard function. Lewis (1981) stated the fact that many standard theoretical distributions, including the two common failure time distributions, the Weibull and the exponential are special cases or limiting cases of the Burr-XII distribution. Evans and Ragab (1983) obtained Bayes estimates for shape parameter and reliability function on type-II censored samples. Mousa (1995) obtained empirical Bayes estimation of the shape parameter and reliability function based on accelerated type-II censored data. Moore and Papadopoulos (2000) obtained bayes estimates for shape parameter and the reliability function when the other shape parameter was assumed to be known. Mousa and Jaheen (2002) obtained bayes approximate estimates for the two parameters and reliability function based on progressive type-II censored samples. Based on the same progressive samples as above, Soliman (2005) obtained the bayes estimate using both the symmetric and

Corresponding Author: Safaa Ali Nasir, Department of Information Technology, Technical College of Management-Baghdad, Foundation of Technical Education, Iraq 
asymmetric loss functions. The usefulness and properties of the Burr-XII distribution as a failure model were discussed in many papers (Al-Hussaini and Jaheen, 1992; Wingo, 1993; Wang et al., 1996). AlKutubi and Ibrahim (2009) carried out a comparison between maximum likelihood estimator and Bayesian estimator of the parameter and survival function for exponential distribution under Jeffrey prior and extension of Jeffrey prior. Ahmed et al. (2010) did comparative study for the maximum likelihood estimator and Bayesian estimators for parameters of weibull distribution under Jeffrey prior and extension of Jeffrey prior information.

The probability density function of two parameter Burr-XII distribution becomes:

$f(t ; \alpha, \beta)=\alpha \beta t^{\alpha-1}\left(1+t^{\alpha}\right)^{-\beta-1} ; t, \alpha, \beta>0$

where, $\alpha, \beta$ are the shape parameters of the distribution. The reliability function is given by:

$$
\mathrm{R}(\mathrm{t})=\operatorname{Pr}[\mathrm{T}>\mathrm{t}]=1-\mathrm{F}(\mathrm{t})=\int_{\mathrm{t}}^{\infty} \mathrm{f}(\mathrm{u} ; \alpha, \beta) \mathrm{du}=\left(1+\mathrm{t}^{\alpha}\right)^{-\beta}
$$

And the hazard function become:

$h(t)=\frac{f(t)}{R(t)}=\frac{\alpha \beta t^{\alpha-1}}{\left(1+t^{\alpha}\right)}$

Which are obviously depend nonmonotonically on failure time.

The rest of the study is arranged as follow. In Methods, maximum likelihood estimator, estimator of method of median, Bayes estimator by using Jeffrey prior information, Bayes estimator by using modified of Jeffrey prior information and Bayes estimator by using extension of Jeffrey prior information are presented. In Results, simulation study is discussed and the results are presented and followed by conclusion.

\section{MATERIALS AND METHODS}

Maximum Likelihood Estimation (MLE): We introduce the concept of maximum likelihood estimation with two parameter Burr-XII distribution. We have set of random failure times $\left(t_{1}, t_{1}, \ldots t_{n}\right)$ and vectors of unknown parameters $\theta=\left(\theta_{1}, \ldots, \theta_{\mathrm{k}}\right)$, then the likelihood function is:

$$
\mathrm{L}(\mathrm{t} ; \theta)=\prod_{\mathrm{i}=1}^{\mathrm{n}} \mathrm{f}\left(\mathrm{t}_{\mathrm{i}} ; \theta\right)
$$

The $\mathrm{i}^{\text {th }}$ elements of the score vector with respect to $\theta$ is:

$\mathrm{U}_{\mathrm{i}}(\theta)=\frac{\partial \ln \mathrm{L}(\mathrm{t}, \theta)}{\partial \theta_{\mathrm{i}}}, \mathrm{i}=1, \ldots, \mathrm{k}$

Now we can find the maximum likelihood estimator by using two parameter Burr-XII distribution with Parameters $\alpha, \beta$. The probability density function of two parameters Burr-XII distributions is given by:

$f(t ; \alpha, \beta)=\alpha \beta t^{\alpha-1}\left(1+t^{\alpha}\right)^{-\beta-1} ; t, \alpha, \beta>0$

The likelihood function is:

$\mathrm{L}\left(\mathrm{t}_{1}, \mathrm{t}_{2}, \ldots, \mathrm{t}_{\mathrm{n}} ; \alpha, \beta\right)=\prod_{\mathrm{i}=1}^{\mathrm{n}} \mathrm{f}\left(\mathrm{t}_{\mathrm{i}} ; \alpha, \beta\right)$

$=\alpha^{\mathrm{n}} \beta^{\mathrm{n}} \prod_{\mathrm{i}=1}^{\mathrm{n}} \mathrm{t}_{\mathrm{i}}^{\alpha-1} \prod_{\mathrm{i}=1}^{\mathrm{n}}\left(1+\mathrm{t}_{\mathrm{i}}^{\alpha}\right)^{-\beta-1}$

The score vector is:

$\mathrm{U}(\theta)=\frac{\partial \ln \mathrm{L}(\mathrm{t} ; \alpha, \beta)}{\partial \beta}=\frac{\mathrm{n}}{\beta}-\sum_{\mathrm{i}=1}^{\mathrm{n}} \log \left(1+\mathrm{t}_{\mathrm{i}}^{\alpha}\right)$

Let $\mathrm{U}(\theta)=0$, then the maximum likelihood estimator is Eq. 1:

$\beta_{\mathrm{m} . \mathrm{le}}^{\wedge}=\frac{\mathrm{n}}{\sum_{\mathrm{i}=1}^{\mathrm{n}} \log \left(1+\mathrm{t}_{\mathrm{i}}^{\alpha}\right)}$

The shape Parameter $\alpha$ is taken to be constant. Since the maximum likelihood estimator is invariant and one to one mapping. The maximum likelihood estimator of Reliability function is Eq. 2:

$\mathrm{R}_{\mathrm{m} .1 . \mathrm{e}}^{\wedge}(\mathrm{t})=\left(1+\mathrm{t}^{\alpha}\right)^{-\beta_{\mathrm{m} .1 . \mathrm{e}}^{\wedge}}$

Method of Median (MOM): We can found quantiles of parametric distribution by:

$q=1-\left(1+t_{q}^{c}\right)^{-\beta}$

Then:

$\mathrm{t}_{\mathrm{q}}=\left[(1-\mathrm{q})^{-1 / \beta}-1\right]^{1 / \mathrm{c}}$

So the median is: 
$\operatorname{median}(\mathrm{t})=\left[0.5^{-1 / \beta}-1\right]^{1 / \mathrm{c}}$

The median estimator is Eq. 3:

$$
\beta_{\text {MOM }}^{\wedge}=\frac{\log (2)}{\log \left(1+\operatorname{median}(t)^{\mathrm{c}}\right)}
$$

The moment estimator of Reliability function is Eq. 4:

$$
\mathbf{R}_{\mathrm{m} . \mathrm{o.m}}^{\wedge}(\mathrm{t})=\left(1+\mathrm{t}^{\alpha}\right)^{-\beta_{\mathrm{m} . \mathrm{m} \cdot \mathrm{m}}^{\wedge}}
$$

Bayes Using Jeffrey prior information (BJ): Let $\left(t_{1}, . . t_{n}\right)$ be a random sample of size $n$ with distribution function $\mathrm{F}(\mathrm{t}, \alpha, \beta)$ and probability density function $\mathrm{f}(\mathrm{t}$, $\alpha, \beta)$. In the two parameter Burr-XII case, we assumed that the probability density function of the lifetime is given by:

$$
f(t ; \alpha, \beta)=\alpha \beta t^{\alpha-1}\left(1+t^{\alpha}\right)^{-\beta-1} ; t, \alpha, \beta>0
$$

We find Jeffery prior by taking:

$$
\mathrm{g}(\beta) \propto \sqrt{\mathrm{I}(\beta)}
$$

Where:

$$
\mathrm{g}(\beta)=\mathrm{k} \sqrt{\mathrm{I}(\beta)}
$$

Then:

$$
\mathrm{g}(\beta)=\mathrm{k} \frac{\sqrt{\mathrm{n}}}{\beta}
$$

with $k$ is a constant and $I(\beta)=-E\left(\frac{\partial^{2} \log L}{\partial \beta^{2}}\right)$ is fisher information.

We can found Bayes estimator with Jeffrey prior of parameter by using posterior distribution which equal to conditional distribution, depend on joint probability density function and marginal probability density function, so the posterior distribution is given by:

$$
\begin{aligned}
& \mathrm{h}\left(\beta \backslash \mathrm{t}_{1}, \mathrm{t}_{2}, \ldots, \mathrm{t}_{\mathrm{n}}\right)=\frac{\mathrm{L}\left(\mathrm{t}_{1}, \mathrm{t}_{2}, \ldots, \mathrm{t}_{\mathrm{n}} ; \alpha, \beta\right) \mathrm{g}(\beta)}{\int_{0}^{\infty} \mathrm{L}\left(\mathrm{t}_{1}, \mathrm{t}_{2}, \ldots, \mathrm{t}_{\mathrm{n}} ; \alpha, \beta\right) \mathrm{g}(\beta) \mathrm{d} \beta} \\
& =\frac{\left[\sum_{\mathrm{i}=1}^{\mathrm{n}} \log \left(1+\mathrm{t}_{\mathrm{i}}^{\alpha}\right)\right]^{\mathrm{n}} \beta^{\mathrm{n}-1} \mathrm{e}^{-\sum_{i=1}^{\mathrm{n}} \log \left(1+\mathrm{t}_{i}^{\alpha}\right) \beta}}{\sqrt{\mathrm{n}}} ; \beta>0
\end{aligned}
$$

where, $\Gamma$. is gamma function.

The above posterior distribution is gamma distribution with parameters $\left(\sum_{\mathrm{i}=1}^{\mathrm{n}} \log \left(1+\mathrm{t}_{\mathrm{i}}^{\alpha}\right), \mathrm{n}\right)$.

By using the well-known squared error loss function, then the Bayes estimator of the scale parameter will represent the expected of the posterior distribution with respect to the parameter Eq. 5:

$\beta_{\mathrm{BJ}}^{\wedge}=\underset{\text { post }}{\mathrm{E}}(\beta)=\int_{0}^{\infty} \beta \mathrm{h}\left(\beta \backslash \mathrm{t}_{1}, \mathrm{t}_{2}, \ldots, \mathrm{t}_{\mathrm{n}}\right) \mathrm{d} \beta=\frac{\mathrm{n}}{\sum_{\mathrm{i}=1}^{\mathrm{n}} \log \left(1+\mathrm{t}_{\mathrm{i}}^{\alpha}\right)}$

By using the same posterior distribution and loss function, we can found the estimator of Reliability function by taking the posterior expected to that function, then Eq. 6:

$$
\left[\sum_{i=1}^{n} \log \left(I+t_{i}^{a}\right)\right]^{n}
$$

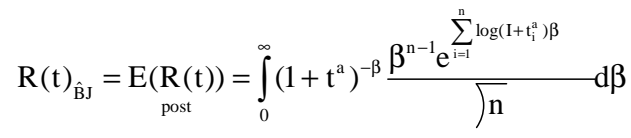

$$
=\left[\frac{\sum_{i=1}^{n} \log \left(1+t_{i}^{\alpha}\right)}{\sum_{i=1}^{n} \log \left(1+t_{i}^{\alpha}\right)+\log \left(1+t^{\alpha}\right)}\right]^{n}
$$

Bayes Using Modify Jeffrey prior information (BMOD): The modify of Jeffery prior is given by:

$$
\mathrm{g}(\beta)=\frac{\mathrm{k}}{\sqrt{\beta^{3}}}
$$

where, $\mathrm{k}$ is a constant.

Let $\left(t_{1}, . . t_{n}\right)$ be a random sample of size $n$ distributed two parameter Burr-XII distribution, then the posterior distribution by using modified Jeffery prior is:

$$
\begin{aligned}
& h\left(\beta \backslash t_{1}, t_{2}, \ldots, t_{n}\right)=\frac{L\left(t_{1}, t_{2}, \ldots, t_{n} ; \alpha, \beta\right) g(\beta)}{\int_{0}^{\infty} L\left(t_{1}, t_{2}, \ldots, t_{n} ; \alpha, \beta\right) g(\beta) d \beta} \\
& =\frac{\left[\sum_{i=1}^{n} \log \left(1+t_{i}^{\alpha}\right)\right]^{n-\frac{1}{2}} \beta^{n-\frac{3}{2}} e^{-\sum_{i=1}^{n} \log \left(1+t_{i}^{\alpha}\right) \beta}}{\sqrt[n]{ }-\frac{1}{2}} ; \beta>0
\end{aligned}
$$

where, $)$. is gamma function. 
The above posterior distribution is gamma distribution with parameters $\left(\sum_{i=1}^{n} \log \left(1+t_{i}^{\alpha}\right), n-\frac{1}{2}\right)$. By using the well-known squared error loss function, then the Bayes estimator of the scale parameter depending on modified Jeffery prior will represent the expected of the posterior distribution with respect to the parameter Eq. 7:

$$
\beta_{\text {BMOD }}^{\wedge}=\underset{\text { post }}{E}(\beta)=\int_{0}^{\infty} \beta h\left(\beta \backslash t_{1}, t_{2}, \ldots, t_{n}\right) d \beta=\frac{n-\frac{1}{2}}{\sum_{i=1}^{n} \log \left(1+t_{i}^{\alpha}\right)}
$$

By using the same posterior distribution and loss function, we can found the Bayes estimator of Reliability function depending on modified Jeffery prior by taking the posterior expected to that function, then Eq. 8:

$$
\begin{aligned}
& \mathrm{R}_{\text {BMOD }}^{\wedge}(\mathrm{t})=\underset{\text { post }}{\mathrm{E}}(\mathrm{R}(\mathrm{t}))= \\
& \int_{0}^{\infty}\left(1+\mathrm{t}^{\alpha}\right)^{-\beta} \frac{\left[\sum_{\mathrm{i}=1}^{\mathrm{n}} \log \left(1+\mathrm{t}_{\mathrm{i}}^{\alpha}\right)\right]^{\mathrm{n}-\frac{1}{2}} \beta^{\mathrm{n}-\frac{3}{2} \mathrm{e}^{-\sum_{i=1}^{n} \log \left(1+\mathrm{t}_{i}^{\alpha}\right) \beta}}}{\sqrt{\mathrm{n}-\frac{1}{2}}} \mathrm{~d} \beta \\
& =\left[\frac{\sum_{\mathrm{i}=1}^{\mathrm{n}} \log \left(1+\mathrm{t}_{\mathrm{i}}^{\alpha}\right)}{\sum_{\mathrm{i}=1}^{\mathrm{n}} \log \left(1+\mathrm{t}_{\mathrm{i}}^{\alpha}\right)+\log \left(1+\mathrm{t}^{\alpha}\right)}\right]^{\mathrm{n}-\frac{1}{2}}
\end{aligned}
$$

Bayes Using Extension Jeffrey prior information (BEXT): The extension of Jeffery prior is taking $\mathrm{g}(\beta) \propto[\mathrm{I}(\beta)]^{\mathrm{c}} ; \mathrm{c} \in \mathrm{R}^{+}$.

Then the extended Jeffery prior Information is:

$$
\mathrm{g}(\beta)=\mathrm{k} \frac{\mathrm{n}^{\mathrm{c}}}{\beta^{2 \mathrm{c}}}
$$

where, $\mathrm{k}$ is a constant.

Let $\left(\mathrm{t}_{1}, . . \mathrm{t}_{\mathrm{n}}\right)$ be a random sample of size $\mathrm{n}$ distributed two parameter Burr-XII distribution, then the posterior distribution by using extended Jeffery prior is:

$$
\begin{aligned}
& h\left(\beta \backslash t_{1}, t_{2}, \ldots, t_{n}\right)=\frac{L\left(t_{1}, t_{2}, \ldots, t_{n} ; \alpha, \beta\right) g(\beta)}{\int_{0}^{\infty} L\left(t_{1}, t_{2}, \ldots, t_{n} ; \alpha, \beta\right) g(\beta) d \beta} \\
& =\frac{\left[\sum_{i=1}^{n} \log \left(1+t_{i}^{\alpha}\right)\right]^{n-2 c+1} \beta^{n-2 c} e^{-\sum_{i=1}^{n} \log \left(1+t_{i}^{\alpha}\right) \beta}}{\sqrt{n-2 c+1}} ; \beta>0
\end{aligned}
$$

where, $)$. is gamma function.

The above posterior distribution is gamma distribution with parameters $\left(\sum_{i=1}^{n} \log \left(1+t_{i}^{\alpha}\right), n-2 c+1\right)$. By using the well-known squared error loss function and then the Bayes estimator of the scale parameter depending on extended Jeffery prior will represent the expected of the posterior distribution with respect to the parameter Eq. 9:

$\beta_{\mathrm{BEXT}}^{\wedge}=\underset{\text { post }}{\mathrm{E}}(\beta)=\int_{0}^{\infty} \beta \mathrm{h}\left(\beta \backslash \mathrm{t}_{1}, \mathrm{t}_{2}, \ldots, \mathrm{t}_{\mathrm{n}}\right) \mathrm{d} \beta=\frac{\mathrm{n}-2 \mathrm{c}+1}{\sum_{\mathrm{i}=1}^{\mathrm{n}} \log \left(1+\mathrm{t}_{\mathrm{i}}^{\alpha}\right)}$

By using the same posterior distribution and loss function, we can found the Bayes estimator of Reliability function depending on extended Jeffery prior by taking the posterior expected to that function, then Eq. 10:

$$
\begin{aligned}
& \mathrm{R}_{\text {BEXT }}^{\wedge}(\mathrm{t})=\underset{\text { post }}{\mathrm{E}}(\mathrm{R}(\mathrm{t}))=\int_{0}^{\infty}\left(1+\mathrm{t}^{\alpha}\right)^{-\beta} \\
& \frac{\left[\sum_{\mathrm{i}=1}^{\mathrm{n}} \log \left(1+\mathrm{t}_{\mathrm{i}}^{\alpha}\right)\right]^{\mathrm{n}-2 \mathrm{c}+1} \beta^{\mathrm{n}-2 \mathrm{c}} \mathrm{e}^{-\sum_{\mathrm{i}=1}^{\mathrm{n} \log \left(1+\mathrm{t}_{\mathrm{i}}^{\alpha}\right) \beta}}}{\sqrt{\mathrm{n}-2 \mathrm{c}+1}} \mathrm{~d} \beta \\
& =\left[\frac{\sum_{\mathrm{i}=1}^{\mathrm{n}} \log \left(1+\mathrm{t}_{\mathrm{i}}^{\alpha}\right)}{\sum_{\mathrm{i}=1}^{\mathrm{n}} \log \left(1+\mathrm{t}_{\mathrm{i}}^{\alpha}\right)+\log \left(1+\mathrm{t}^{\alpha}\right)}\right]^{\mathrm{n}-2 \mathrm{c}+1}
\end{aligned}
$$

\section{RESULTS}

In this simulation study, we have chosen $n=10,20$, 50 and 100 to represent small, moderate and large sample size, several values of parameter $\beta=0.5,1.5$ and $\alpha=$ $0.5,1$, three values of Jeffery extension $c=0.5,1,1.5$. The number of replication used was $(\mathrm{L}=1000)$. The simulation program was written by using matlab-R2010b program. After the Reliability function was estimated, Integrated Mean Square Error (IMSE) and Integrated Mean Absolute Percentage Error (IMAPE) was calculated to compare the methods of estimation, where:

$$
\begin{aligned}
& \operatorname{IMSE}\left(R^{\wedge}(t)\right)=\frac{1}{L} \sum_{i=1}^{L}\left\{\frac{1}{n_{t}} \sum_{j=1}^{n_{t}}\left(R_{i}\left(t_{j}\right)-R_{i}^{\wedge}\left(t_{j}\right)\right)^{2}\right\} \\
& =\frac{1}{L} \sum_{i=1}^{L} \operatorname{MSE}\left(R_{i}^{\wedge}(t)\right) \\
& \operatorname{IMAPE}\left(R^{\wedge}(t)\right)=\frac{1}{L} \sum_{i=1}^{L}\left\{\frac{1}{n_{t}} \sum_{j=1}^{n_{i}}\left|\frac{R_{i}\left(t_{j}\right)-R_{i}^{\wedge}\left(t_{j}\right)}{R_{i}\left(t_{j}\right)}\right|\right\} \\
& =\frac{1}{L} \sum_{i=1}^{L} \operatorname{MAPE}\left(R_{i}^{\wedge}(t)\right)
\end{aligned}
$$


J. Math. \& Stat., 8 (1): 42-48, 2012

Table 1: IMSE for different estimators of reliability function for Burr-IXX distribution

\begin{tabular}{|c|c|c|c|c|c|c|c|c|}
\hline $\mathrm{N}$ & $\beta$ & $\mathrm{a}$ & $\mathrm{C}$ & MLE & MOM & BJ & BMOD & BEXT \\
\hline \multirow[t]{12}{*}{10} & 0.5 & 0.5 & 0.5 & 0.097299 & 0.167476 & 0.088000 & 0.082966 & 0.088000 \\
\hline & & & 1.0 & 0.097299 & 0.167476 & 0.088000 & 0.082966 & 0.089303 \\
\hline & & & 1.5 & 0.097299 & 0.167476 & 0.088000 & 0.082966 & 0.099744 \\
\hline & & 1 & 0.5 & 0.109796 & 0.168739 & 0.098155 & 0.095252 & 0.098155 \\
\hline & & & 1.0 & 0.109796 & 0.168739 & 0.098155 & 0.095252 & 0.095661 \\
\hline & & & 1.5 & 0.109796 & 0.168739 & 0.098155 & 0.095252 & 0.118096 \\
\hline & 1.5 & 0.5 & 0.5 & 0.098030 & 0.171669 & 0.094301 & 0.104755 & 0.094301 \\
\hline & & & 1 & 0.098030 & 0.171669 & 0.094301 & 0.104755 & 0.118083 \\
\hline & 1.5 & & & 0.098030 & 0.171669 & 0.094301 & 0.104755 & 0.182929 \\
\hline & & 1 & 0.5 & 0.076238 & 0.134354 & 0.078898 & 0.089701 & 0.078898 \\
\hline & & & 1 & 0.076238 & 0.134354 & 0.078898 & 0.089701 & 0.092957 \\
\hline & & & 1.5 & 0.076238 & 0.134354 & 0.078898 & 0.089701 & 0.159864 \\
\hline \multirow[t]{12}{*}{20} & 0.5 & 0.5 & 0.5 & 0.045497 & 0.082678 & 0.043338 & 0.042148 & 0.043338 \\
\hline & & 1 & & 0.045497 & 0.082678 & 0.043338 & 0.042148 & 0.040778 \\
\hline & & & 1.5 & 0.045497 & 0.082678 & 0.043338 & 0.042148 & 0.044115 \\
\hline & & 1 & 0.5 & 0.052386 & 0.101753 & 0.049719 & 0.049279 & 0.049719 \\
\hline & & & 1.0 & 0.052386 & 0.101753 & 0.049719 & 0.049279 & 0.050604 \\
\hline & & & 1.5 & 0.052386 & 0.101753 & 0.049719 & 0.049279 & 0.056396 \\
\hline & 1.5 & 0.5 & 0.5 & 0.054685 & 0.106592 & 0.053015 & 0.055546 & 0.053015 \\
\hline & & & 1.0 & 0.054685 & 0.106592 & 0.053015 & 0.055546 & 0.057592 \\
\hline & & & 1.5 & 0.054685 & 0.106592 & 0.053015 & 0.055546 & 0.070165 \\
\hline & & 1 & 0.5 & 0.037490 & 0.069048 & 0.037434 & 0.039644 & 0.037434 \\
\hline & & & 1.0 & 0.037490 & 0.069048 & 0.037434 & 0.039644 & 0.045676 \\
\hline & & & 1.5 & 0.037490 & 0.069048 & 0.037434 & 0.039644 & 0.057584 \\
\hline \multirow[t]{12}{*}{50} & 0.5 & 0.5 & 0.5 & 0.017670 & 0.035176 & 0.017218 & 0.016784 & 0.017218 \\
\hline & & & 1.0 & 0.017670 & 0.035176 & 0.017218 & 0.016784 & 0.015435 \\
\hline & & & 1.5 & 0.017670 & 0.035176 & 0.017218 & 0.016784 & 0.018199 \\
\hline & & 1 & 0.5 & 0.021995 & 0.044474 & 0.021540 & 0.021460 & 0.021540 \\
\hline & & & 1.0 & 0.021995 & 0.044474 & 0.021540 & 0.021460 & 0.020571 \\
\hline & & & 1.5 & 0.021995 & 0.044474 & 0.021540 & 0.021460 & 0.020546 \\
\hline & 1.5 & 0.5 & 0.5 & 0.021147 & 0.040454 & 0.021061 & 0.021624 & 0.021061 \\
\hline & & & 1.0 & 0.021147 & 0.040454 & 0.021061 & 0.021624 & 0.023359 \\
\hline & & & 1.5 & 0.021147 & 0.040454 & 0.021061 & 0.021624 & 0.025962 \\
\hline & & 1 & 0.5 & 0.015866 & 0.031604 & 0.015957 & 0.016397 & 0.015957 \\
\hline & & & 1.0 & 0.015866 & 0.031604 & 0.015957 & 0.016397 & 0.017018 \\
\hline & & & 1.5 & 0.015866 & 0.031604 & 0.015957 & 0.016397 & 0.020372 \\
\hline \multirow[t]{12}{*}{100} & 0.5 & 0.5 & 0.5 & 0.008174 & 0.017864 & 0.008084 & 0.008012 & 0.008084 \\
\hline & & & 1.0 & 0.008174 & 0.017864 & 0.008084 & 0.008012 & 0.007939 \\
\hline & & & 1.5 & 0.008174 & 0.017864 & 0.008084 & 0.008012 & 0.007802 \\
\hline & & 1 & 0.5 & 0.010985 & 0.022510 & 0.010848 & 0.010794 & 0.010848 \\
\hline & & & 1.0 & 0.010985 & 0.022510 & 0.010848 & 0.010794 & 0.010137 \\
\hline & & & 1.5 & 0.010985 & 0.022510 & 0.010848 & 0.010794 & 0.011117 \\
\hline & 1.5 & 0.5 & 0.5 & 0.011304 & 0.024118 & 0.011230 & 0.011341 & 0.011230 \\
\hline & & & 1.0 & 0.011304 & 0.024118 & 0.011230 & 0.011341 & 0.011502 \\
\hline & & & 1.5 & 0.011304 & 0.024118 & 0.011230 & 0.011341 & 0.011704 \\
\hline & & 1 & 0.5 & 0.008746 & 0.017002 & 0.008784 & 0.008910 & 0.008784 \\
\hline & & & 1.0 & 0.008746 & 0.017002 & 0.008784 & 0.008910 & 0.008245 \\
\hline & & & 1.5 & 0.008746 & 0.017002 & 0.008784 & 0.008910 & 0.009008 \\
\hline
\end{tabular}

Table 2: IMAPE for different estimators of reliability function for Burr-IXX distribution

\begin{tabular}{|c|c|c|c|c|c|c|c|c|}
\hline sample size & $\beta$ & a & $\mathrm{C}$ & MLE & MOM & BJ & BMOD & BEXT \\
\hline \multirow[t]{12}{*}{10} & 0.5 & 0.5 & 0.5 & 1.218688 & 1.613908 & 1.171048 & 1.160971 & 1.171048 \\
\hline & & & 1.0 & 1.218688 & 1.613908 & 1.171048 & 1.160971 & 1.229474 \\
\hline & & & 1.5 & 1.218688 & 1.613908 & 1.171048 & 1.160971 & 1.343347 \\
\hline & & 1 & 0.5 & 1.573683 & 1.975610 & 1.500355 & 1.500331 & 1.500355 \\
\hline & & & 1.0 & 1.573683 & 1.975610 & 1.500355 & 1.500331 & 1.531491 \\
\hline & & & 1.5 & 1.573683 & 1.975610 & 1.500355 & 1.500331 & 1.756645 \\
\hline & 1.5 & 0.5 & 0.5 & 3.304435 & 4.480305 & 3.219003 & 3.407876 & 3.219003 \\
\hline & & & 1.0 & 3.304435 & 4.480305 & 3.219003 & 3.407876 & 3.661916 \\
\hline & & & 1.5 & 3.304435 & 4.480305 & 3.219003 & 3.407876 & 4.697723 \\
\hline & & 1 & 0.5 & 4.581081 & 6.140895 & 4.761742 & 5.220388 & 4.761742 \\
\hline & & & 1.0 & 4.581081 & 6.140895 & 4.761742 & 5.220388 & 5.412519 \\
\hline & & & 1.5 & 4.581081 & 6.140895 & 4.761742 & 5.220388 & 7.646040 \\
\hline \multirow[t]{3}{*}{20} & 0.5 & 0.5 & 0.5 & 0.831333 & 1.145455 & 0.815355 & 0.811434 & 0.815355 \\
\hline & & & 1.0 & 0.831333 & 1.145455 & 0.815355 & 0.811434 & 0.808963 \\
\hline & & & 1.5 & 0.831333 & 1.145455 & 0.815355 & 0.811434 & 0.871535 \\
\hline
\end{tabular}




\begin{tabular}{|c|c|c|c|c|c|c|c|c|}
\hline & & 1 & 0.5 & 1.101116 & 1.541479 & 1.076221 & 1.079078 & 1.076221 \\
\hline & & & 1.0 & 1.101116 & 1.541479 & 1.076221 & 1.079078 & 1.112382 \\
\hline & & & 1.5 & 1.101116 & 1.541479 & 1.076221 & 1.079078 & 1.198813 \\
\hline & 1.5 & 0.5 & 0.5 & 2.453089 & 3.511775 & 2.411506 & 2.475372 & 2.411506 \\
\hline & & & 1.0 & 2.453089 & 3.511775 & 2.411506 & 2.475372 & 2.514138 \\
\hline & & & 1.5 & 2.453089 & 3.511775 & 2.411506 & 2.475372 & 2.808121 \\
\hline & & 1 & 0.5 & 3.174063 & 4.417894 & 3.200505 & 3.343299 & 3.200505 \\
\hline & & & 1.0 & 3.174063 & 4.417894 & 3.200505 & 3.343299 & 3.716205 \\
\hline & & & 1.5 & 3.174063 & 4.417894 & 3.200505 & 3.343299 & 4.277705 \\
\hline \multirow[t]{12}{*}{50} & 0.5 & 0.5 & 0.5 & 0.540878 & 0.757795 & 0.534980 & 0.530325 & 0.534980 \\
\hline & & & 1.0 & 0.540878 & 0.757795 & 0.534980 & 0.530325 & 0.505597 \\
\hline & & & 1.5 & 0.540878 & 0.757795 & 0.53498 & 0.530325 & 0.553460 \\
\hline & & 1 & 0.5 & 0.711198 & 1.027467 & 0.705198 & 0.705933 & 0.705198 \\
\hline & & & 1.0 & 0.711198 & 1.027467 & 0.705198 & 0.705933 & 0.702752 \\
\hline & & & 1.5 & 0.711198 & 1.027467 & 0.705198 & 0.705933 & 0.713449 \\
\hline & 1.5 & 0.5 & 0.5 & 1.525529 & 2.140118 & 1.527982 & 1.553385 & 1.527982 \\
\hline & & & 1.0 & 1.525529 & 2.140118 & 1.527982 & 1.553385 & 1.592026 \\
\hline & & & 1.5 & 1.525529 & 2.140118 & 1.527982 & 1.553385 & 1.706960 \\
\hline & & 1 & 0.5 & 2.082806 & 2.965990 & 2.090612 & 2.130048 & 2.090612 \\
\hline & & & 1.0 & 2.082806 & 2.965990 & 2.090612 & 2.130048 & 2.211407 \\
\hline & & & 1.5 & 2.082806 & 2.965990 & 2.090612 & 2.130048 & 2.471071 \\
\hline \multirow[t]{12}{*}{100} & 0.5 & 0.5 & 0.5 & 0.368788 & 0.542103 & 0.366779 & 0.365148 & 0.366779 \\
\hline & & & 1.0 & 0.368788 & 0.542103 & 0.366779 & 0.365148 & 0.360615 \\
\hline & & & 1.5 & 0.368788 & 0.542103 & 0.366779 & 0.365148 & 0.355581 \\
\hline & & 1 & 0.5 & 0.502793 & 0.741205 & 0.500341 & 0.500192 & 0.500341 \\
\hline & & & 1.0 & 0.502793 & 0.741205 & 0.500341 & 0.500192 & 0.489976 \\
\hline & & & 1.5 & 0.502793 & 0.741205 & 0.500341 & 0.500192 & 0.512076 \\
\hline & 1.5 & 0.5 & 0.5 & 1.103021 & 1.630775 & 1.097325 & 1.101767 & 1.097325 \\
\hline & & & 1.0 & 1.103021 & 1.630775 & 1.097325 & 1.101767 & 1.124927 \\
\hline & & & 1.5 & 1.103021 & 1.630775 & 1.097325 & 1.101767 & 1.147173 \\
\hline & & 1 & 0.5 & 1.544966 & 2.178792 & 1.563636 & 1.583782 & 1.563636 \\
\hline & & & 1.0 & 1.544966 & 2.178792 & 1.563636 & 1.583782 & 1.507385 \\
\hline & & & 1.5 & 1.544966 & 2.178792 & 1.563636 & 1.583782 & 1.579497 \\
\hline
\end{tabular}

The results of the simulation study are summarized and tabulated in Table 1 and 2 of the five estimators for all sample size and $(\alpha, \beta, c)$ values respectively.

In each row of Table 1 and 2 we have five values of estimators that is the maximum likelihood, method of median, Bayes using Jeffery prior, Bayes using modified Jeffery and Bayes using extended Jeffery prior. The best method is the method that gives smallest value of (IMSE) and (IMAPE).

\section{DISCUSSION}

In Table 1 and 2, when we compared reliability estimation for Burr-XXI distribution by using mentioned methods we find the best estimator is Bayes using modified Jeffery prior estimator (BMOD) for small values of $\beta$ and maximum likelihood for large values and this result is true for all values of parameter and sample sizes used in the study.

\section{CONCLUSION}

The estimated Reliability function of Burr-XXI distribution from modified bayes estimation is the best compared for small values of $\beta$ and maximum likelihood method for large values of $\beta$. The maximum likelihood method give better result than method of median. The method of median give worst result than other methods. When the number of sample size increases the Integrated Mean Square Error (IMSE) and Integrated Mean Square Error (IMAPE) decreases in all cases.

\section{REFERANCES}

Ahmed, A.O.M., H.S. Al-Kutubi and N.A. Ibrahim, 2010. Comparison of the Bayesian and Maximum Likelihood Estimation for Weibull distribution. J. Math. Stat., 6: 100-104. DOI: 10.3844/jmssp.2010.100.104

Al-Hussaini, E.K. and Z.F. Jaheen, 1992. Bayesian estimation of the parameters, reliability and failure rate functions of the burr type xii failure model. J. Stat. Comput. Simul., 41: 31-40. DOI: 10.1080/00949659208811389

AL-Kutubi, H.S. and N.A. Ibrahim, 2009. On the estimation of survival function and parameter exponential life time distribution. J. Math. Stat., 5: 130-135. DOI: $10.3844 /$ jmssp.2009.130.135 
Burr, I.W., 1942. Cumulative frequency functions. Ann. Math. Stat., 13: 215-232. DOI: 10.1214/aoms/1177731607

Evans, I.G. and A.S. Ragab, 1983. Bayesian inferences given a type- ${ }^{2}$ censored sample from a burr distribution. Commun. Stat.-Theor. Math., 12: 1569-1580. DOI: 10.1080/03610928308828551

Lewis, A.W., 1981. The Burr Distribution as a General Parametric Family in Survivorship and Reliability Theory Applications. 1st Edn., Department of Biostatistics, Chapel Hill, N.C., pp: 148.

Moore, D. and A.S. Papadopoulos, 2000. The Burr Type XII distribution as a failure Model under various loss functions. Microelect. Reliab., 40: 2117-2122. DOI: 10.1016/S0026-2714(00)00031-7

Mousa, M.A.M.A., 1995. Empirical Bayes estimators for the burr type XII accelerated life testing model based on type- 2 censored data. J. Stat. Comput. Simul., 52: 95-103. DOI: $10.1080 / 00949659508811655$
Mousa, M.A.M.A. and Z.F. Jaheen, 2002. Statistical inference for the Burr model based on progressively censored data. Comput. Math. Appli., 43: 1441-1449. DOI: 10.1016/S08981221(02)00110-4

Soliman, A.A., 2005. Estimation of parameters of life from progressively censored data using Burr-XII model. IEEE. Trans. Reliab., 54: 34-42. DOI: 10.1109/TR.2004.842528

Wang, F.K., J.B. Keats and W.J. Zimmer, 1996. Maximum likelihood estimation of the Burr XII parameters with censored and uncensored data. Microelect. Reliabl., 36: 359-362. DOI: 10.1016/0026-2714(95)00077-1

Wingo, D.R., 1993. Maximum likelihood estimation of Burr XII distribution parameters under Type II censoring. Microelect. Reliabl., 33: 1251-1257. DOI: 10.1016/0026-2714(93)90126-J 